\title{
A Riemann-Hillbert approach to Painlevé IV
}

Marius van der Put, Jaap Top

To cite this article: Marius van der Put, Jaap Top (2013) A Riemann-Hilbert approach to Painlevé IV, Journal of Nonlinear Mathematical Physics 20: Supplement 1, 165-177, DOI: https://doi.org/10.1080/14029251.2013.862442

To link to this article: https://doi.org/10.1080/14029251.2013.862442

Published online: 04 January 2021 


\title{
A Riemann-Hilbert approach to Painlevé IV
}

\author{
Marius van der Put and Jaap Top \\ Johann Bernoulli Institute, University of Groningen \\ P.O.Box 407, 9700 AK Groningen, the Netherlands \\ mvdput@math.rug.nl j.top@rug.nl \\ Received 11 April 2012 \\ Accepted 5 september 2012
}

\begin{abstract}
The methods of [vdP-Sa, vdP1, vdP2] are applied to the fourth Painlevé equation. One obtains a RiemannHilbert correspondence between moduli spaces of rank two connections on $\mathbb{P}^{1}$ and moduli spaces for the monodromy data. The moduli spaces for these connections are identified with Okamoto-Painlevé varieties and the Painlevé property follows. For an explicit computation of the full group of Bäcklund transformations, rank three connections on $\mathbb{P}^{1}$ are introduced, inspired by the symmetric form for PIV, studied by M. Noumi and Y. Yamada.
\end{abstract}

Keywords: Moduli space for linear connections, Irregular singularities, Stokes matrices, Monodromy spaces, Isomonodromic deformations, Painlevé equations

2000 Mathematics Subject Classification: 14D20, 14D22, 34M55

\section{Introduction}

In this paper we apply the methods of [vdP-Sa, vdP1, vdP2] to the fourth Painlevé equation. We refer only to a few items of the extensive literature on Okamoto-Painlevé varieties. More details on Stokes matrices and the analytic classification of singularities can be found in [vdP-Si].

The Riemann-Hilbert approach to the Painlevé equation PIV consists of the construction of a moduli space $\mathscr{M}$ of connections on the projective line and a moduli space $\mathscr{R}$ for the monodromy data. The Riemann-Hilbert morphism $R H: \mathscr{M} \rightarrow \mathscr{R}$ assigns to a connection its monodromy data. The fibres of $R H$, i.e., the isomonodromic families in $\mathscr{M}$, are parametrized by $t \in T=\mathbb{C}$. The explicit form of the fibres produces the solutions of PIV.

$R H^{\text {ext }}: \mathscr{M}^{+}\left(\theta_{0}, \theta_{\infty}\right) \rightarrow \mathscr{R}^{+}\left(\theta_{0}, \theta_{\infty}\right) \times T$, the extended Riemann-Hilbert morphism, is an analytic isomorphism between rather subtle moduli spaces $\mathscr{M}^{+}\left(\theta_{0}, \theta_{\infty}\right)$ and $\mathscr{R}^{+}\left(\theta_{0}, \theta_{\infty}\right) \times T$, depending on parameters $\theta_{0}, \theta_{\infty}$ and provided with a level structure (or parabolic structure). The Painlevé Property for PIV with parameters $\theta_{0}, \theta_{\infty}$ follows from this as well as the identification of $\mathscr{M}^{+}\left(\theta_{0}, \theta_{\infty}\right)$ with an Okamoto-Painlevé variety. Formulas for Bäcklund transformations, rational and Riccati solutions for PIV are derived.

The construction of $\mathscr{M}$ involves the choice of a set $\mathbf{S}$ of differential modules over $\mathbb{C}(z)$. In the first part of this paper the 'classical' choice for $\mathbf{S}$ is treated. The second choice for $\mathbf{S}$ is inspired by the symmetric form for PIV [No, No-Y], studied by M. Noumi and Y. Yamada. This leads to a different construction of $\mathscr{M}, \mathscr{R}$ and Okamoto-Painlevé varieties, treated in $\S 3$.

\section{The classical choice for $S$ and $\mathscr{R}$}

Let $\mathbf{S}$ be the set of the isomorphy classes of the differential modules $\left(M, \delta_{M}\right)$ over $\mathbb{C}(z)$ (with $\left.\delta_{M}(f m)=\left(z \frac{d}{d z} f\right) m+f \delta_{M}(m)\right)$ having the properties:

$\operatorname{dim} M=2 ; \Lambda^{2} M$ is trivial; $0, \infty$ are the singular points and the Katz invariants are $r(0)=0, r(\infty)=2$. 
The variable $z$ is normalized such that the (generalized) eigenvalues at $\infty$ are $\pm\left(z^{2}+\frac{t}{2} z\right)$. Finally, we exclude the case that $M$ is a direct sum of two proper submodules since this situation does not produce solutions for PIV.

The monodromy data at $\infty$ are given by the matrices

$$
\left(\begin{array}{cc}
\alpha & 0 \\
0 & \frac{1}{\alpha}
\end{array}\right),\left(\begin{array}{cc}
1 & 0 \\
a_{1} & 1
\end{array}\right),\left(\begin{array}{cc}
1 & a_{2} \\
0 & 1
\end{array}\right),\left(\begin{array}{cc}
1 & 0 \\
a_{3} & 1
\end{array}\right),\left(\begin{array}{cc}
1 & a_{4} \\
0 & 1
\end{array}\right)
$$

with respect to a basis of the symbolic solution space $V(\infty)$ at $z=\infty$ corresponding to the direct sum expression $V(\infty)=V_{z^{2}+\frac{t}{2} z} \oplus V_{-\left(z^{2}+\frac{t}{2} z\right)}=\mathbb{C} e_{1} \oplus \mathbb{C} e_{2}$. The first matrix is the formal monodromy and the others are the four Stokes matrices. The topological monodromy to $p_{\infty}$ at $z=\infty$ (which equals the topological monodromy at $z=0$ ) is the product of these matrices in this order. Further we exclude the case $a_{1}=a_{2}=a_{3}=a_{4}=0$, since this corresponds to the direct sum situation. The monodromy data form a variety $\mathscr{A}:=\mathbb{C}^{*} \times\left(\mathbb{C}^{4} \backslash\{(0,0,0,0)\}\right)$.

The base change $e_{1}, e_{2} \mapsto \lambda e_{1}, \lambda^{-1} e_{2}$ induces an action of $\mathbb{G}_{m}$ on $\mathscr{A}$. The monodromy space $\mathscr{R}$ is the quotient $\mathscr{A} / \mathbb{G}_{m}$. This quotient can be obtained by gluing the subspaces $\mathscr{R}_{j}, j=1, \ldots, 4$ of $\mathscr{A}$, defined by $a_{j}=1$.

We observe (see [vdP-Sa], Theorem 1.7) that the map $\mathbf{S} \rightarrow \mathscr{R} \times T$, which maps a module in $\mathbf{S}$ to its monodromy data and the value of $t \in T=\mathbb{C}$, is bijective.

The parameter space is $\mathscr{P}=\mathbb{C} \times \mathbb{C}^{*}$ and $\mathscr{R} \rightarrow \mathscr{P}$ maps an element of $\mathscr{R}$ to $\left(\right.$ trace $\left(\right.$ top $\left.\left._{\infty}\right), \alpha\right)$. The fibre above $(s, \alpha)$ is denoted by $\mathscr{R}[s, \alpha]$. This fibre is a smooth, connected surface for $s \neq \pm 2$. The fibre $\mathscr{R}[2, \alpha]$ has one singular point and this point corresponds to to $p_{\infty}=\left(\begin{array}{ll}1 & 0 \\ 0 & 1\end{array}\right)$. Similarly, $\mathscr{R}[-2, \alpha]$ has one singular point corresponding to to $p_{\infty}=-\left(\begin{array}{ll}1 & 0 \\ 0 & 1\end{array}\right)$.

The singular points are the reason for introducing a level structure (or 'parabolic structure' in the terminology of [Bo, In, IIS1, IIS2, IISA]). For the monodromy data this is a line $L \subset V(\infty)$ which is invariant under $t o p_{\infty}$. The new monodromy space is denoted by $\mathscr{R}^{+}$. For a module $M$ in $\mathbf{S}$ the level structure is a 1-dimensional submodule $N$ of $\mathbb{C}((z)) \otimes M$. The submodule $N$ corresponds to an eigenvector of the topological monodromy to $p_{0}$ at $z=0$ (which is equal to $t o p_{\infty}$ ). The new set is denoted by $\mathbf{S}^{+}$. For the parameter space, the level structure is the introduction of an eigenvalue $\beta$ of top $_{\infty}$. The new parameter space $\mathscr{P}^{+}=\mathbb{C}^{*} \times \mathbb{C}^{*}$ maps to $\mathscr{P}$ by $(\beta, \alpha) \mapsto\left(\beta+\beta^{-1}, \alpha\right)$.

The fibres of $\mathscr{R}^{+} \rightarrow \mathscr{P}^{+}$are denoted by $\mathscr{R}^{+}(\beta, \alpha)$. The morphism $\mathscr{R}^{+}(\beta, \alpha) \rightarrow \mathscr{R}\left[\beta+\beta^{-1}, \alpha\right]$ is an isomorphism for $\beta \neq \pm 1$. A computation shows that

Lemma 1.1. $\mathscr{R}^{+}( \pm 1, \alpha) \rightarrow \mathscr{R}[ \pm 2, \alpha]$ is the minimal resolution.

The map $\mathbf{S}^{+} \rightarrow \mathscr{P}^{+}$is defined by $\beta=e^{2 \pi i \lambda}$ where $\delta_{M} n=\lambda n$ for a basis vector $n$ of $N \subset$ $\mathbb{C}((z)) \otimes M$ and $\alpha$ as before. The fibre is written as $\mathbf{S}^{+}(\beta, \alpha)$.

Lemma 1.2. The map $\mathbf{S}^{+}(\beta, \alpha) \rightarrow \mathscr{R}^{+}(\beta, \alpha) \times T$ is bijective.

The reducible locus of $\mathscr{R}^{+}$(i.e., the monodromy data is reducible) is the disjoint union of the closed sets defined (in the notation of $\mathscr{A}$ ) by $a_{2}=a_{4}=0$ and $a_{1}=a_{3}=0$. The space $\mathscr{R}^{+}(\beta, \alpha)$ contains no reducible elements for $\beta^{ \pm 1} \neq \alpha$. If $\beta^{ \pm 1}=\alpha$, then the reducible locus of $\mathscr{R}^{+}(\beta, \alpha)$ consists of two non intersecting projective lines. 


\section{The moduli space $\mathscr{M}\left(\theta_{0}, \theta_{\infty}\right)$}

Choose $\theta_{0}$ with $\beta=e^{\pi i \theta_{0}}$ and $\theta_{\infty}$ with $\alpha=e^{\pi i \theta_{\infty}}$. The aim is to replace the set $\mathbf{S}^{+}(\beta, \alpha)$ by a moduli space of connections $\mathscr{M}\left(\theta_{0}, \theta_{\infty}\right)$ and to study the extended Riemann-Hilbert map $R H^{\text {ext }}$ : $\mathscr{M}\left(\theta_{0}, \theta_{\infty}\right) \rightarrow \mathscr{R}^{+}(\beta, \alpha) \times T$.

Let a module $(M, N) \in \mathbf{S}^{+}(\beta, \alpha)$ be given. We define a connection $(\mathscr{W}, \nabla)$ on the projective line with $\nabla: \mathscr{W} \rightarrow \Omega([0]+3[\infty]) \otimes \mathscr{W}$, with generic fibre $M$, by prescribing the connection $D:=\nabla_{z \frac{d}{d z}}$

locally at $z=0$ as $z \frac{d}{d z}+\left(\begin{array}{cc}\frac{\theta_{0}}{2} & * \\ 0 & -\frac{\theta_{0}}{2}\end{array}\right)$ and locally at $z=\infty$ as $z \frac{d}{d z}+\left(\begin{array}{cc}\omega & 0 \\ 0 & -\omega\end{array}\right)$ with $\omega=z^{2}+\frac{t}{2} z+\frac{\theta_{\infty}}{2}$. This is equivalently to choosing 'invariant lattices' at $z=0$ and $z=\infty$. The invariant lattice at $z=0$ is $\mathbb{C}[[z]] g_{1}+\mathbb{C}[[z]] g_{2} \subset \mathbb{C}((z)) \otimes M$ with $N=\mathbb{C}((z)) g_{1}$ and the matrix of $\delta_{M}$ with respect to $g_{1}, g_{2}$ is $\left(\begin{array}{cc}\frac{\theta_{0}}{2} & * \\ 0 & -\frac{\theta_{0}}{2}\end{array}\right)$. The invariant lattice at $z=\infty$ is $\mathbb{C}\left[\left[z^{-1}\right]\right] h_{1}+\mathbb{C}\left[\left[z^{-1}\right]\right] h_{2} \subset \mathbb{C}\left(\left(z^{-1}\right)\right) \otimes M$ such that $\delta_{M} h_{1}=\omega h_{1}, \delta_{M}^{2} h_{2}=-\omega h_{2}$.

The second exterior power of $(\mathscr{W}, \nabla)$ is $d: O \rightarrow \Omega$. Thus $\mathscr{W}$ has degree 0 and type $O(k) \oplus O(-k)$ with $k \geq 0$. If $M$ is irreducible, then $k \in\{0,1\}$. The reducible modules are studied in Observations 2.2.

We consider the case $k \in\{0,1\}$. The connection $(\mathscr{V}, \nabla)$, defined by replacing the invariant lattice $\mathbb{C}[[z]] g_{1}+\mathbb{C}[[z]] g_{2}$ by $\mathbb{C}[[z]] g_{1}+\mathbb{C}[[z]] z g_{2}$, has type $O \oplus O(-1)$. Further we identify $\mathscr{V}$ with $O e_{1} \oplus O(-[0]) e_{2}$.

\subsection{The connections on $\mathscr{V}:=O e_{1}+O(-[0]) e_{2}$}

The connection $D=\nabla_{z \frac{d}{d z}}: \mathscr{V} \rightarrow O(2[\infty]) \otimes \mathscr{V}$, obtained from $(M, N) \in \mathbf{S}^{+}(\beta, \alpha)$ and the prescribed invariant lattices, has, with respect to the basis $e_{1}, e_{2}$, the matrix $\left(\begin{array}{cc}a & b \\ c & -a\end{array}\right)$ with $a=a_{0}+a_{1} z+a_{2} z^{2}$, $b=b_{-1} z^{-1}+\cdots+b_{2} z^{2}, c=c_{1} z+c_{2} z^{2}$. The local data at $z=\infty$ yields the equations

$$
a_{2}^{2}+b_{2} c_{2}=1,2 a_{1} a_{2}+b_{2} c_{1}+b_{1} c_{2}=t, 2 a_{0} a_{2}+a_{1}^{2}+b_{1} c_{1}+b_{0} c_{2}=\theta_{\infty}+\frac{t^{2}}{4} .
$$

For $z=0$ one obtains $a_{0}\left(a_{0}-1\right)+b_{-1} c_{1}=\frac{\theta_{0}}{2}\left(\frac{\theta_{0}}{2}-1\right)$.

As a start, we forget the level structure $N$ of the pair $(M, N) \in \mathbf{S}^{+}(\beta, \alpha)$ and we assume that $c_{1} z+c_{2} z^{2} \neq 0$. The above variables $a_{*}, b_{*}, c_{*}, t$ and equations define a space $\mathscr{C}$ of dimension 6 . We have to divide by the group $G$ of transformations $e_{1} \mapsto e_{1}, e_{2} \mapsto \lambda e_{2}+\left(x_{0}+x_{1} z^{-1}\right) e_{1}$ of $\mathscr{V}$. The quotient $\mathscr{C} / G$ is by definition the moduli space $\mathscr{M}\left(\theta_{0}, \theta_{\infty}\right)$.

Proposition 2.1. The moduli space $\mathscr{M}\left(\theta_{0}, \theta_{\infty}\right)$ is a good geometric quotient of $\mathscr{C}$ in the sense that there exists a G-equivariant isomorphism $G \times \mathscr{M}\left(\theta_{0}, \theta_{\infty}\right) \rightarrow \mathscr{C}$.

$\mathscr{M}\left(\theta_{0}, \theta_{\infty}\right)$ is smooth for $\theta_{0} \neq 1$. For a connection $D \in \mathscr{M}\left(1, \theta_{\infty}\right)$, which is a singular point, there is a basis of $\widehat{\mathscr{V}}_{0}$ for which $D$ has the form $z \frac{d}{d z}+\left(\begin{array}{cc}\frac{1}{2} & 0 \\ 0 & \frac{1}{2}\end{array}\right)$.

Proof. The 'first standard form' $S T_{1}$ is the closed subset of $\mathscr{C}$ defined by:

$z \frac{d}{d z}+\left(\begin{array}{c}a_{2} z^{2} \\ z+c_{2} z^{2}\end{array}-a_{2} z^{2}\right)$ with $b_{2}=-c_{2}\left(\theta_{\infty}+\frac{t^{2}}{4}-b_{0} c_{2}\right)+t, b_{1}=\theta_{\infty}+\frac{t^{2}}{4}-b_{0} c_{2}, b_{-1}=\frac{\theta_{0}}{2}\left(\frac{\theta_{0}}{2}-1\right)$ and $a_{2}^{2}+c_{2} t-c_{2}^{2}\left(\theta_{\infty}+\frac{t^{2}}{4}-b_{0} c_{2}\right)-1=0$. The obvious morphism $G \times S T_{1} \rightarrow\left\{\left(a_{*}, b_{*}, c_{*}\right) \in \mathscr{C} \mid c_{1} \neq 0\right\}$ is an isomorphism. 
The coordinate ring of $S T_{1}$ is $\mathbb{C}\left[a_{2}, c_{2}, t, b_{0}\right] /\left(a_{2}^{2}+c_{2} t-c_{2}^{2}\left(\theta_{\infty}+\frac{t^{2}}{4}-b_{0} c_{2}\right)-1\right)$ and $S T_{1}$ is nonsingular.

The 'second standard form' $S T_{2}$ is the closed subset of $\mathscr{C}$ defined by:

$z \frac{d}{d z}+\left(\begin{array}{c}a_{0} b \\ c_{1} z+z^{2}-a_{0}\end{array}\right)$ with $b=z^{2}+b_{1} z+b_{0}+b_{-1} z^{-1}, c=c_{1} z+z^{2}$ and $c_{1}+b_{1}=t, b_{1} c_{1}+b_{0}=\theta_{\infty}+\frac{t^{2}}{4}$, $a_{0}\left(a_{0}-1\right)+b_{-1} c_{1}=\frac{\theta_{0}}{2}\left(\frac{\theta_{0}}{2}-1\right)$. The obvious morphism $G \times S T_{2} \rightarrow\left\{\left(a_{*}, b_{*}, c_{*}\right) \in \mathscr{C} \mid c_{2} \neq 0\right\}$ is an isomorphism.

The coordinate ring of $S T_{2}$ is $\mathbb{C}\left[a_{0}, c_{1}, t, b_{-1}\right] /\left(a_{0}\left(a_{0}-1\right)+b_{-1} c_{1}-\frac{\theta_{0}}{2}\left(\frac{\theta_{0}}{2}-1\right)\right)$. For fixed $t$, one finds one singular point: $a_{0}=1 / 2, b_{-1}=c_{1}=0, \theta_{0}=1$.

In the above case, one easily verifies that $D$ has the form $z \frac{d}{d z}+\left(\begin{array}{cc}\frac{1}{2} & 0 \\ 0 & \frac{1}{2}\end{array}\right)$ w.r.t. a basis of $\widehat{\mathscr{V}}_{0}$. The quotient $\mathscr{C} / G$ is obtained by gluing the two 'charts' $S T_{1}$ and $S T_{2}$ in the obvious way.

Observations 2.1 The level structure for $\mathscr{M}\left(\theta_{0}, \theta_{\infty}\right)$.

For a connection $D \in \mathscr{M}\left(\theta_{0}, \theta_{\infty}\right)$, the level structure is a 1-dimensional submodule $N \subset \mathbb{C}((z)) \otimes \widehat{\mathcal{V}}_{0}$ with a generator $n$ such that $\delta n=e^{\pi i \theta_{0}} n$. The space $\mathscr{M}^{+}\left(\theta_{0}, \theta_{\infty}\right)$ denotes the addition of this level structure to $\mathscr{M}\left(\theta_{0}, \theta_{\infty}\right)$.

If $t o p_{0}$, the topological monodromy at $z=0$ of the connection $D$, is not $\pm\left(\begin{array}{ll}1 & 0 \\ 0 & 1\end{array}\right)$, then the level structure $N$ is unique.

If top $_{0}= \pm\left(\begin{array}{ll}1 & 0 \\ 0 & 1\end{array}\right)$, then $\theta_{0} \in \mathbb{Z}$. Further $\widehat{\mathscr{V}_{0}}$ has a basis $v_{1}, v_{2}$ for which $D$ obtains the matrix form $z \frac{d}{d z}+\left(\begin{array}{cc}\frac{\theta_{0}}{2} & 0 \\ 0 & 1-\frac{\theta_{0}}{2}\end{array}\right)$. If $\frac{\theta_{0}}{2} \neq 1-\frac{\theta_{0}}{2}$, the basis $v_{1}, v_{2}$ is unique up to multiplication by constants. Then one defines the level structure $N$ by $N=\mathbb{C}((z)) v_{1}$.

In the final case top $p_{0}=-\left(\begin{array}{ll}1 & 0 \\ 0 & 1\end{array}\right)$ and $\theta_{0}=1$, the connection $D$ does not prescribe a level structure. We replace $\mathscr{M}\left(1, \theta_{\infty}\right)$ by $\mathscr{M}^{+}\left(1, \theta_{\infty}\right)$ defined as the closed subspace of $\mathscr{M}\left(1, \theta_{\infty}\right) \times \mathbb{P}^{1}$ consisting of the equivalence classes of the tuples $(D, L)$ with $D \in \mathscr{M}\left(1, \theta_{\infty}\right)$ and $L$ a line in $\widehat{\mathscr{V}}_{0}$ at $z=0$, invariant under $D$. We will verify that (for fixed $t) \mathscr{M}^{+}\left(1, \theta_{\infty}\right) \rightarrow \mathscr{M}\left(1, \theta_{\infty}\right)$ is the minimal resolution.

Verification. The chart $S T_{2}$ of $\mathscr{M}\left(1, \theta_{\infty}\right)$ consists of the differential operators $z \frac{d}{d z}+\left(\begin{array}{cc}a_{0} & z b \\ c_{1}+z & 1-a_{0}\end{array}\right)$ with

$$
b=z^{2}+b_{1} z+b_{0}+b_{-1} z^{-1}, c_{1}+b_{1}=t, b_{1} c_{1}+b_{0}=\theta_{\infty}+\frac{t^{2}}{4}, a_{0}\left(a_{0}-1\right)+b_{-1} c_{1}=-\frac{1}{4} .
$$

The line $L=\mathbb{C}\left(\begin{array}{l}x_{1} \\ x_{0}\end{array}\right)$ is generated by a nonzero element $\left(\begin{array}{l}x_{1} \\ x_{0}\end{array}\right) \in \mathbb{C}[[z]]^{2}$ satisfying the equation

$$
\left\{z \frac{d}{d z}+\left(\begin{array}{cc}
a_{0} & z b \\
c_{1}+z & 1-a_{0}
\end{array}\right)\right\}\left(\begin{array}{l}
x_{1} \\
x_{0}
\end{array}\right)=\frac{1}{2}\left(\begin{array}{l}
x_{1} \\
x_{0}
\end{array}\right) .
$$

In the case $a_{0}=\frac{1}{2}, b_{-1}=c_{1}=0$, the operator $z \frac{d}{d z}+\left(\begin{array}{cc}a_{0} & z b \\ c_{1}+z & 1-a_{0}\end{array}\right)$ is equivalent over $\mathbb{C}[[z]]$ to $z \frac{d}{d z}+\left(\begin{array}{cc}\frac{1}{2} & 0 \\ 0 & \frac{1}{2}\end{array}\right)$. Thus the possible lines $L$ form a projective line. In the opposite case, the operator is equivalent over $\mathbb{C}[[z]]$ to $z \frac{d}{d z}+\left(\begin{array}{cc}\frac{1}{2} & 1 \\ 0 & \frac{1}{2}\end{array}\right)$ and there is only one $L$.

Observations 2.2 The reducible locus of $\mathscr{M}\left(\theta_{0}, \theta_{\infty}\right)$.

Put $\omega=z^{2}+\frac{t}{2} z+\frac{\theta_{\infty}}{2}$ and let $c \in \mathbb{C}\left[z^{-1}, z\right]$. If a reducible connection is present in $\mathscr{M}\left(\theta_{0}, \theta_{\infty}\right)$, then $\frac{\theta_{0}}{2} \in \pm \frac{\theta_{\infty}}{2}+\mathbb{Z}$. There are two types of reducible modules in $\mathbf{S}^{+}$. Type (1) is represented by $z \frac{d}{d z}+\left(\begin{array}{cc}-\omega & 0 \\ c & \omega\end{array}\right)$ and Type (2) is represented by $z \frac{d}{d z}+\left(\begin{array}{cc}\omega & 0 \\ c & -\omega\end{array}\right)$. 
For a given reducible module $M$, say of type (1), one defines (as before) the connection $(\mathscr{V}, D)$ with generic fibre $M$ by the local operators $z \frac{d}{d z}+\left(\begin{array}{cc}\frac{\theta_{0}}{2} & * \\ 0 & 1-\frac{\theta_{0}}{2}\end{array}\right)$ at $z=0$ and $z \frac{d}{d z}+\left(\begin{array}{cc}\omega & 0 \\ 0 & -\omega\end{array}\right)$ at $z=\infty$. Assume that type (1) is not present in $\mathscr{M}\left(\theta_{0}, \theta_{\infty}\right)$. Then $\mathscr{V} \cong O^{2}(k) \oplus O(-k-1)$ with $k \geq 1$ and one identifies $\mathscr{V}$ with $O(k[0]) e_{1} \oplus O(-(k+1)[0]) e_{2}$. A computation of $D$ in this case leads to two possible relations, namely $\frac{\theta_{0}}{2}=\frac{\theta_{\infty}}{2}-k$ or $1-\frac{\theta_{0}}{2}=\frac{\theta_{\infty}}{2}-k$. Thus one finds the list for type (1). The list for type (2) is found in a similar way.

Type (1) is present in precisely the following cases:

$\theta_{0} \geq \theta_{\infty}$ for $\frac{\theta_{0}}{2} \in \frac{\theta_{\infty}}{2}+\mathbb{Z}$ and $\frac{\theta_{0}}{2} \notin-\frac{\theta_{\infty}}{2}+\mathbb{Z}$

$\theta_{0} \leq-\theta_{\infty}+2$ for $\frac{\theta_{0}}{2} \notin \frac{\theta_{\infty}}{2}+\mathbb{Z}$ and $\frac{\theta_{0}}{2} \in-\frac{\theta_{\infty}}{2}+\mathbb{Z}$

$\theta_{0} \geq \theta_{\infty}$ or $\theta_{0} \leq-\theta_{\infty}+2$ for $\frac{\theta_{0}}{2} \in \frac{\theta_{\infty}}{2}+\mathbb{Z}$ and $\frac{\theta_{0}}{2} \in-\frac{\theta_{\infty}}{2}+\mathbb{Z}$

Type (2) is present in precisely the following cases:

$\theta_{0} \leq \theta_{\infty}+2$ for $\frac{\theta_{0}}{2} \in \frac{\theta_{\infty}}{2}+\mathbb{Z}$ and $\frac{\theta_{0}}{2} \notin-\frac{\theta_{\infty}}{2}+\mathbb{Z}$

$\theta_{0} \geq-\theta_{\infty}$ for $\frac{\theta_{0}}{2} \notin \frac{\theta_{\infty}}{2}+\mathbb{Z}$ and $\frac{\theta_{0}}{2} \in-\frac{\theta_{\infty}}{2}+\mathbb{Z}$

$\theta_{0} \leq \theta_{\infty}+2$ or $\theta_{0} \geq-\theta_{\infty}$ for $\frac{\theta_{0}}{2} \in \frac{\theta_{\infty}}{2}+\mathbb{Z}$ and $\frac{\theta_{0}}{2} \in-\frac{\theta_{\infty}}{2}+\mathbb{Z}$

Examples: We use the notation $z \frac{d}{d z}+\left(\begin{array}{cc}a & b \\ c & -a\end{array}\right)$ of the space $\mathscr{C}$. Suppose $b=0$.

Then $a_{2}^{2}=1,2 a_{1} a_{2}=t, 2 a_{0} a_{2}+a_{1}^{2}=\theta_{\infty}+\frac{t^{2}}{4},\left(a_{0}-\frac{1}{2}\right)^{2}=\left(\frac{\theta_{0}}{2}-\frac{1}{2}\right)^{2}$ and the non zero element $c_{1} z+c_{2} z^{2}$ is unique up to multiplication by a non zero constant. One finds in general four reducible families (with some overlap for $\theta_{0}=1$ and/or $\theta_{\infty}= \pm 1$ ):

$b=0, \quad a_{2}=1, \quad a_{1}=\frac{t}{2}, \quad a_{0}=\frac{\theta_{\infty}}{2}, \quad \frac{\theta_{\infty}}{2}=\frac{1}{2} \pm\left(\frac{\theta_{0}}{2}-\frac{1}{2}\right)$ and

$b=0, a_{2}=-1, a_{1}=-\frac{t}{2}, a_{0}=-\frac{\theta_{\infty}}{2},-\frac{\theta_{\infty}}{2}=\frac{1}{2} \pm\left(\frac{\theta_{0}}{2}-\frac{1}{2}\right)$.

We observe that these examples are precisely the cases of an equality sign in the lists for type (1) and type (2).

Proposition 2.2. Put $\beta=e^{\pi i \theta_{0}}, \alpha=e^{\pi i \theta_{\infty}}$. Let $F: \mathscr{M}^{+}\left(\theta_{0}, \theta_{\infty}\right) \rightarrow \mathbf{S}^{+}(\beta, \alpha)$ be the map that sends $a$ tuple $(D, L)$ to $(M, N)$ where $M$ is the generic fibre of $D$ and $N=\mathbb{C}((z)) \otimes L$. The map $F$ is injective and its image contains the 'irreducible locus' of $\mathbf{S}^{+}(\beta, \alpha)$. A component of the 'reducible locus' lies in the image of $F$ if and only if $\theta_{0}, \theta_{\infty}$ satisfy the corresponding inequality of Observations 2.2.

Proof. The injectivity of $F$ follows from the construction of $\mathscr{M}^{+}\left(\theta_{0}, \theta_{\infty}\right)$. If $M$ is irreducible then the vector bundle $\mathscr{W}$, introduced in the beginning of this section, has type $O(k) \oplus O(-k)$ for some $k \in\{0,1\}$. Therefore the subbundle $\mathscr{V}$ has type $O \oplus O(-1)$ and can be identifies with $O e_{1} \oplus O(-[0]) e_{2}$. Thus the image of $F$ contains the 'irreducible locus' of $\mathbf{S}^{+}(\beta, \alpha)$. The final statement follows from Observations 2.2.

Define $\mathbf{S}^{+}\left(\theta_{0}, \theta_{\infty}\right) \subset \mathbf{S}^{+}(\beta, \alpha)$ (for $\beta=e^{\pi i \theta_{0}}, \alpha=e^{\pi i \theta_{\infty}}$ ) to be the image of $F$ and let $\mathscr{R}^{+}\left(\theta_{0}, \theta_{\infty}\right) \subset \mathscr{R}^{+}(\beta, \alpha)$ be the corresponding open subset.

Corollary 2.1. $R H^{\text {ext }}: \mathscr{M}^{+}\left(\theta_{0}, \theta_{\infty}\right) \rightarrow \mathscr{R}^{+}\left(\theta_{0}, \theta_{\infty}\right) \times T$, the extended Riemann-Hilbert map, is an analytic isomorphism.

Proof. $R H^{\text {ext }}$ is bijective since $\mathbf{S}^{+}\left(\theta_{0}, \theta_{\infty}\right) \rightarrow \mathscr{R}^{+}\left(\theta_{0}, \theta_{\infty}\right) \times T$ is bijective. The two spaces $\mathscr{M}^{+}\left(\theta_{0}, \theta_{\infty}\right)$ and $\mathscr{R}^{+}\left(\theta_{0}, \theta_{\infty}\right) \times T$ are smooth and so $R H^{\text {ext }}$ is an analytic isomorphism (see [vdP1, vdP2]). 


\subsection{Isomonodromic families, Okamoto-Painlevé spaces}

An isomonodromic family above the chart $S_{2}$ of $\mathscr{M}^{+}\left(\theta_{0}, \theta_{\infty}\right)$ has the form $z \frac{d}{d z}+A$ with $A=$ $\left(\begin{array}{cc}a_{0} & b \\ c & -a_{0}\end{array}\right)$ with $c=z^{2}-q z, b=z^{2}+b_{1} z+b_{0}+b_{-1} z^{-1}, b=z^{2}+(t+q) z+q(t+q)+\theta_{\infty}+\frac{t^{2}}{4}+$ $\left(\frac{\left(a_{0}-\frac{1}{2}\right)^{2}-\left(\frac{\theta_{0}}{2}-\frac{1}{2}\right)^{2}}{q}\right) z^{-1}$, where $a_{0}$ and $q$ are functions of $t$. Isomonodromy is equivalent to the existence of an operator $\frac{d}{d t}+B$, commuting with $z \frac{d}{d z}+A$. In other terminology $z \frac{d}{d z}+A, \frac{d}{d t}+B$ is a Lax pair. This is equivalent to the equation $\frac{d}{d t}(A)=z \frac{d}{d z}(B)+[A, B]$. One observes that $B$ has trace zero and that the entries of $B$ have the form $d_{-1}(t) z+d_{0}(t)+d_{1}(t) z$. Using MAPLE one obtains the solution $B=\left(\begin{array}{ll}0 & B_{1} \\ B_{2} & 0\end{array}\right)$ with $B_{1}=\frac{q\left(q+b_{1}\right)+b_{0}}{2} z^{-1}+\frac{b_{1}+q}{2}+\frac{1}{2} z, B_{2}=\frac{1}{2} z$,

$$
\begin{gathered}
q^{\prime}=a_{0}-\frac{1}{2}, a_{0}^{\prime}=\frac{b_{-1}+q\left(q\left(b_{1}+q\right)+b_{0}\right)}{2} \text { and the fourth Painlevé equation } \\
q^{\prime \prime}=\frac{\left(q^{\prime}\right)^{2}}{2 q}+\frac{3 q^{3}}{2}+t q^{2}+\frac{q}{8}\left(4 \theta_{\infty}+t^{2}\right)-\frac{\left(\theta_{0}-1\right)^{2}}{8 q} \text { with parameters } \theta_{0}, \theta_{\infty} .
\end{gathered}
$$

Isomonodromy for reducible families. An isomonodromic family of operators $z \frac{d}{d z}+\left(\begin{array}{cc}\omega & 0 \\ z^{2}-q z & -\omega\end{array}\right)$, with $\omega=z^{2}+\frac{t}{2} z+\frac{d}{2}$, commutes with an operator of the form $\frac{d}{d t}+\left(\begin{array}{cc}\tau & 0 \\ \frac{z}{2} & -\tau\end{array}\right)$. One computes that $\tau=\frac{2 z+2 q+t}{4}$ and $q^{\prime}=q^{2}+\frac{t}{2} q+\frac{d-1}{2}$. Then $q$ is a Riccati solution of PIV with $d=\theta_{\infty}$ and $d=1 \pm\left(\theta_{0}-1\right)$.

An isomonodromic family $z \frac{d}{d z}+\left(\begin{array}{cc}-\omega 0 \\ z^{2}-q z & 0\end{array}\right)$ with $\omega=z^{2}+\frac{t}{2} z+\frac{d}{2}$ produces the differential equation $q^{\prime}=-q^{2}-\frac{t}{2} q-\frac{d+1}{2}$. Then $q$ is a Riccati solution of PIV with $d=\theta_{\infty}$ and $d=-1 \pm\left(\theta_{0}-1\right)$.

Observations 2.3 The solutions $q_{r}$ with $r \in \mathscr{R}^{+}\left(\theta_{0}, \theta_{\infty}\right)$.

The fibre of $\mathscr{M}^{+}\left(\theta_{0}, \theta_{\infty}\right) \rightarrow \mathscr{R}^{+}\left(\theta_{0}, \theta_{\infty}\right)$ above $r$ is, by Corollary 2.1 , isomorphic to $T$. Write $q_{r}$ for the function $q$ appearing in the formula for the chart $S T_{2}$. Then $q_{r}$ is a meromorphic solution of PIV, defined on all of $T$.

Theorem 2.1. The fourth Painlevé equation has the Painlevé property. The moduli space $\mathscr{M}^{+}\left(\theta_{0}, \theta_{\infty}\right)$ is analytically isomorphic to the Okamoto-Painlevé space for PIV with parameters $\theta_{0}, \theta_{\infty}$.

Proof. Let a local solution $Q$ of PIV with parameters $\theta_{0}, \theta_{\infty}$ be given. Let $U$ be an open disk, where $Q$ is holomorphic and has no zeros. Consider the operator $z \frac{d}{d z}+\left(\begin{array}{cc}\tilde{a}_{0} & \tilde{b} \\ z^{2}-Q z-\tilde{a}_{0}\end{array}\right)$ with $\tilde{a}_{0}=\frac{d Q}{d t}+\frac{1}{2}$, and $\tilde{b}=z^{2}+(t+Q) z+Q(t+Q)+\theta_{\infty}+\frac{t^{2}}{4}+\frac{\left(\tilde{a}_{0}-\frac{1}{2}\right)^{2}-\left(\frac{\theta_{0}}{2}-\frac{1}{2}\right)^{2}}{Q} z^{-1}$. This defines an analytic map $U \rightarrow \mathscr{M}^{+}\left(\theta_{0}, \theta_{\infty}\right)$. Since $Q$ is a local solution of PIV, the map $U \rightarrow \mathscr{M}^{+}\left(\theta_{0}, \theta_{\infty}\right) \rightarrow \mathscr{R}^{+}\left(\theta_{0}, \theta_{\infty}\right)$ is constant. Let $r$ be its image. Then $Q$ coincides with $q_{r}$ on $U$. Thus $Q$ extends to a global solution of PIV and this equation has the Painlevé property.

The bundle $\mathscr{M}^{+}\left(\theta_{0}, \theta_{\infty}\right) \rightarrow T$, with its foliation defined by the fibres of the morphism $\mathscr{M}^{+}\left(\theta_{0}, \theta_{\infty}\right) \rightarrow \mathscr{R}^{+}\left(\theta_{0}, \theta_{\infty}\right)$, is the Okamoto-Painlevé variety according to the isomorphism of Corollary 2.1.

We note that $\mathscr{R}^{+}\left(\theta_{0}, \theta_{\infty}\right)$ is the space of initial conditions. 


\subsection{Aut $\left(\mathbf{S}^{+}\right)$and Bäcklund transformations}

Natural automorphisms of $\mathbf{S}^{+}$are:

(1). $\sigma_{1}:(M, N) \mapsto\left(M, N^{*}\right)$ where $N^{*}$ is a submodule of $\mathbb{C}((z)) \otimes M$ such that $N \oplus N^{*}=\mathbb{C}((z)) \otimes M$. This is well defined for $\beta \neq \pm 1$. For $\beta= \pm 1$, the module $N^{*}$ might not exist or might not be unique. It seems correct to define $N^{*}:=N$ for $\beta= \pm 1$.

(2). $\sigma_{2}:(M, N) \mapsto(M \otimes A, N \otimes A)$, where $A=\mathbb{C}((z)) a$ and $\delta a=\frac{1}{2} a$.

(3). $\sigma_{3}:(M, N) \mapsto \mathbb{C}(z) \otimes_{\phi}(M, N)$, where $\phi$ is the automorphism of $\mathbb{C}(z)$ which is the identity on $\mathbb{C}$ and maps $z$ to $i z$. Let $\operatorname{Aut}\left(\mathbf{S}^{+}\right)$denote the group generated by $\sigma_{j}, j=1,2,3$.

\begin{tabular}{|c|c|c|c|c|}
\hline & $\beta$ & $\alpha$ & $t$ & $z$ \\
\hline$\sigma_{1}$ & $\beta^{-1}$ & $\alpha$ & $t$ & $z$ \\
\hline$\sigma_{2}$ & $-\beta$ & $-\alpha$ & $t$ & $z$ \\
\hline$\sigma_{3}$ & $\beta$ & $\alpha^{-1}$ & $i t$ & $i z$ \\
\hline
\end{tabular}

The above group is commutative and has order 16 . The following table is a choice of lifting the generators to actions on $\theta_{0}, \theta_{\infty}, t, z$.

\begin{tabular}{|c|c|c|c|c|}
\hline & $\theta_{0}$ & $\theta_{\infty}$ & $t$ & $z$ \\
\hline$\tilde{\sigma}_{1}$ & $2-\theta_{0}$ & $\theta_{\infty}$ & $t$ & $z$ \\
\hline$\tilde{\sigma}_{2}$ & $\theta_{0}+1$ & $\theta_{\infty}+1$ & $t$ & $z$ \\
\hline$\tilde{\sigma}_{3}$ & $\theta_{0}$ & $-\theta_{\infty}$ & $i t$ & $i z$ \\
\hline
\end{tabular}

The induced morphisms $\tilde{\sigma}_{1}: \mathscr{M}^{+}\left(\theta_{0}, \theta_{\infty}\right) \rightarrow \mathscr{M}^{+}\left(-\theta_{0}+2, \theta_{\infty}\right)$ and $\tilde{\sigma}_{3}: \mathscr{M}^{+}\left(\theta_{0}, \theta_{\infty}\right) \rightarrow$ $\mathscr{M}^{+}\left(\theta_{0},-\theta_{\infty}\right)$ are evident from the standard operators representing the points of $\mathscr{M}^{+}\left(\theta_{0}, \theta_{\infty}\right)$. A MAPLE computation yields the explicit morphisms $\tilde{\sigma}_{2}: \mathscr{M}^{+}\left(\theta_{0}, \theta_{\infty}\right) \rightarrow \mathscr{M}^{+}\left(\theta_{0}+1, \theta_{\infty}+1\right)$. The formulas are given with respect to the coordinates $a=a_{0}, q$ of an open subset (namely $q \neq 0$ in the chart $S T_{2}$ ) of the first space and $\tilde{a}=\tilde{a}_{0}, \tilde{q}$ of an open subset of the second space. The assumption that the operator $z \frac{d}{d z}+A\left(a, q, \theta_{0}, \theta_{\infty}, z\right)$, belonging to $\mathscr{M}^{+}\left(\theta_{0}, \theta_{\infty}\right)$, is equivalent, by a transformation of the type $U_{-2} z^{-2}+U_{-1} z^{-1}+U_{0}+U_{1} z+U_{2} z^{2}$, to the operator $z \frac{d}{d z}+A\left(\tilde{a}, \tilde{q}, \theta_{0}+1, \theta_{\infty}+1, z\right)$, belonging to $\mathscr{M}^{+}\left(\theta_{0}+1, \theta_{\infty}+1\right)$, leads to the following formulas

$$
\begin{gathered}
\tilde{q}=\frac{-4 q^{2} \theta_{\infty}+4 a^{2}-4 q^{3} t-q^{2} t^{2}-4 q^{4}-4 q^{2} \theta_{0}+\theta_{0}^{2}-4 a \theta_{0}}{4 q\left(q t-\theta_{0}+2 a+2 q^{2}\right)} \\
\tilde{a}=\frac{\text { long }}{16 q^{2}\left(q t-\theta_{0}+2 a+2 q^{2}\right)^{2}} .
\end{gathered}
$$

The substitution $a=q^{\prime}+\frac{1}{2}$ in the first formula produces $\tilde{q}$ in terms of $q, q^{\prime}$ and the parameters $\theta_{0}, \theta_{\infty}$, this is the Bäcklund transformation in terms of solutions. The second formula is obtained by substitution $\tilde{a}=\tilde{q}^{\prime}+\frac{1}{2}$ and an expression for $\tilde{q}^{\prime}$ coming from the first formula and the equation for $q^{\prime \prime}$.

The term $q t-\theta_{0}+2 a+2 q^{2}$ in the denominator of the formulas indicates that the morphism $\tilde{\sigma}_{3}$ is in general a rational equivalence and is not defined on leaves of the foliation with $a=q^{\prime}+\frac{1}{2}$ and $q^{\prime}+q^{2}+\frac{t}{2} q+\frac{-\theta_{0}+1}{2}=0$. This occurs precisely when $\theta_{0}=-\theta_{\infty}$ and the reducible locus of $\mathscr{M}^{+}\left(\theta_{0}, \theta_{\infty}\right)$ is not present in the corresponding $\mathscr{M}^{+}\left(1+\theta_{0}, 1+\theta_{\infty}\right)$ (compare Observations 2.2 and the Riccati equations for reducible families). 
We note that the group $<\tilde{\sigma}_{1}, \tilde{\sigma}_{2}, \tilde{\sigma}_{3}>$ contains the two shifts $\theta_{0} \mapsto \theta_{0}+2, \theta_{\infty} \mapsto \theta_{\infty}$ and $\theta_{0} \mapsto \theta_{0}, \theta_{\infty} \mapsto \theta_{\infty}+2$. One observes that, in comparison with the book of Gromak et al. [Gr] and Okamoto's paper [O3], there is still a missing generator for the group of all Bäcklund transformations of PIV. This generator does not seem to come from a 'natural' automorphism of $\mathbf{S}^{+}$(i.e., constructions of linear algebra for differential modules and operations with the differential field $\mathbb{C}(z)$ ). In the final section we will investigate another set of differential modules $\mathbf{S}$, inspired by Noumi's symmetric form of PIV ( [No, No-Y]). As is shown by Noumi, this will easily produce all Bäcklund transformations and moreover all rational and Riccati solutions.

\section{The Noumi-Yamada family}

M. Noumi and Y. Yamada produced a $3 \times 3$-Lax pair, arising from the Lax formalism of the modified KP hierarchy, for the symmetric form of PIV, namely

$$
\begin{gathered}
z \frac{d}{d z}+\left(\begin{array}{ccc}
\varepsilon_{1} & f_{1} & 1 \\
z & \varepsilon_{2} & f_{2} \\
f_{0} z & z & \varepsilon_{3}
\end{array}\right), \frac{d}{d t}+\left(\begin{array}{ccc}
-q_{1} & 1 & 0 \\
0 & -q_{2} & 1 \\
z & 0 & -q_{3}
\end{array}\right) \text {, leading to equations } \\
\varepsilon_{1}^{\prime}=\varepsilon_{2}^{\prime}=\varepsilon_{3}^{\prime}=0 ; f_{1}-f_{2}=-q_{1}+q_{3} ; f_{2}-f_{0}=q_{1}-q_{2} ; f_{0}-f_{1}=q_{2}-q_{3}, \\
f_{0}^{\prime}=f_{0}\left(f_{1}-f_{2}\right)+\left(1-\varepsilon_{1}+\varepsilon_{3}\right) ; f_{1}^{\prime}=f_{1}\left(f_{2}-f_{0}\right)+\left(\varepsilon_{1}-\varepsilon_{2}\right) ; f_{2}^{\prime}=f_{2}\left(f_{0}-f_{1}\right)+\left(\varepsilon_{2}-\varepsilon_{3}\right) .
\end{gathered}
$$

Since the local exponents $\varepsilon_{*}$ at $z=0$ are constants in an isomonodromic family, we can and will suppose $\varepsilon_{1}+\varepsilon_{2}+\varepsilon_{3}=0$. Then we may and will also suppose that $q_{1}+q_{2}+q_{3}=0$. Further it is assumed that $t=f_{0}+f_{1}+f_{2}$.

Then $f_{1}$ satisfies the fourth Painlevé equation $y^{\prime \prime}=\frac{\left(y^{\prime}\right)^{2}}{2 y}+\frac{3}{2} y^{3}-2 t y^{2}+\left(\frac{t^{2}}{2}+\theta_{\infty}\right) y-\frac{\left(\theta_{0}-1\right)^{2}}{2 y}$ with $\theta_{0}=1+\varepsilon_{1}-\varepsilon_{2}$, and $\theta_{\infty}=1+\varepsilon_{1}-\varepsilon_{3}$. After rescaling $t \mapsto \frac{t}{\sqrt{2}}, y \mapsto-\sqrt{2} y$ one obtains 'our' equation $y^{\prime \prime}=\frac{\left(y^{\prime}\right)^{2}}{2 y}+\frac{3}{2} y^{3}+t y^{2}+\left(t^{2}+4 \theta_{\infty}\right) \frac{y}{8}-\frac{\left(\theta_{0}-1\right)^{2}}{8 y}$.

Using this symmetric form one finds the extended Weyl group of $A_{2}$ as group of Bäcklund transformations. For example $\pi:\left(f_{0}, f_{1}, f_{2}\right) \mapsto\left(f_{1}, f_{2}, f_{0}\right)$ translates into the 'missing' Bäcklund transformation of $\S 2.3$, namely

$\theta_{0} \mapsto-\frac{1}{2} \theta_{0}+\frac{1}{2} \theta_{\infty}+2, \quad \theta_{\infty} \mapsto-\frac{3}{2} \theta_{0}-\frac{1}{2} \theta_{\infty}+2$.

This is the inspiration for the new class $\mathbf{S}$ of differential modules $M$ over $\mathbb{C}(z)$, defined by: $\operatorname{dim} M=3 ; \Lambda^{3} M$ is trivial; the only singular points are $0, \infty ; 0$ is regular singular and the Katz invariant of $\infty$ is $\frac{2}{3}$. After scaling the variable $z$ the generalized eigenvalues at $\infty$ are:

$q_{0}=z^{2 / 3}+\frac{t}{3} z^{1 / 3}, q_{1}=\zeta^{2} z^{2 / 3}+\zeta \frac{t}{3} z^{1 / 3}, q_{2}=\zeta z^{2 / 3}+\zeta^{2} \frac{t}{3} z^{1 / 3}$ where $\zeta=e^{2 \pi i / 3}$.

Invariant lattices at $z=\infty$. For $M \in \mathbf{S}$ the operator $D=\nabla_{z \frac{d}{d z}}$ has at $z=\infty$ has the form $z \frac{d}{d z}+\operatorname{diag}\left(q_{0}, q_{1}, q_{2}\right)$ with respect to a basis $e_{0}, e_{1}, e_{2}$. A lattice $\Lambda$ at $z=\infty$ is called invariant if $z^{-1} D(\Lambda) \subset \Lambda$. We respect to the basis $h_{0}=e_{0}+e_{1}+e_{2}, h_{1}=z^{1 / 3}\left(e_{0}+\zeta e_{1}+\zeta^{2} e_{2}\right)$, and $h_{2}=z^{-1 / 3}\left(e_{0}+\zeta^{2} e_{1}+\zeta e_{2}\right), D$ has the form $z \frac{d}{d z}+\left(\begin{array}{ccc}0 & z & \frac{t}{3} \\ \frac{t}{3} & \frac{1}{3} & 1 \\ z & \frac{t}{3} z & -\frac{1}{3}\end{array}\right)$. Thus $\Lambda_{0}:=<h_{0}, h_{1}, h_{2}>$ is an invariant lattice. $\Lambda_{1}:=<h_{0}, z^{-1} h_{1}, h_{2}>$ is the only invariant lattice of codimension 1 in $\Lambda_{0}$ 
and the operator $D$ has with respect to this basis the form $z \frac{d}{d z}+\left(\begin{array}{ccc}0 & 1 & \frac{t}{3} \\ \frac{t}{3} z-\frac{2}{3} & z \\ z & \frac{t}{3} & -\frac{1}{3}\end{array}\right)$. The invariant lattice $\Lambda_{2}:=<z^{-1} h_{0}, z^{-1} h_{1}, h_{2}>$ has codimension 2 in $\Lambda_{0}$. All invariant lattices at $z=\infty$ are $\left\{z^{n} \Lambda_{i} \mid n \in \mathbb{Z}, i=0,1,2\right\}$.

The Noumi-Yamada Lax pair has one additional feature, namely: there exists $U \in \mathrm{GL}(3, \mathbb{C}[[z]])$ with $U=1+U_{1} z+U_{2} z^{2}+\ldots$ such that

$$
U^{-1}\left\{z \frac{d}{d z}+\left(\begin{array}{ccc}
\varepsilon_{1} & f_{1} & 1 \\
z & \varepsilon_{2} & f_{2} \\
f_{0} z & z & \varepsilon_{3}
\end{array}\right)\right\} U=z \frac{d}{d z}+\left(\begin{array}{ccc}
\varepsilon_{1} & * & * \\
0 & \varepsilon_{2} & * \\
0 & 0 & \varepsilon_{3}
\end{array}\right) \text {, with all } * \in \mathbb{C}
$$

Level structure for $\mathbf{S}$. This leads to a 'level structure' or 'parabolic structure' for the elements $M \in \mathbf{S}$ consisting of differential submodules $M_{1} \subset M_{2} \subset \mathbb{C}((z)) \otimes M$ of dimensions 1 and 2 over $\mathbb{C}((z))$. Let $\mathbf{S}^{+}$denote the set of the differential modules in $\mathbf{S}$, provided with a level structure.

The moduli space $\mathscr{R}$ for the analytic data.

The singular directions $d$ for $q_{k}-q_{\ell}$ are computed as follows. $z \frac{d}{d z}(y)=\left(q_{k}-q_{\ell}\right) y$ has solution $\exp \left(\frac{3}{2}\left(\zeta^{2 k}-\zeta^{2 \ell}\right) z^{2 / 3}+3\left(\zeta^{k}-\zeta^{\ell}\right) z^{1 / 3}\right)$. Write $z=e^{i d}$ and $\zeta^{2 k}-\zeta^{2 \ell}=\left|\zeta^{2 k}-\zeta^{2 \ell}\right| e^{i \phi(k, \ell)}$. Then $\left|y\left(r e^{i d}\right)\right|$ has maximal descent for $r \rightarrow \infty$ if and only if $\frac{2}{3} d+\phi(k, \ell)=\pi+\mathbb{Z} 2 \pi$ (equivalently, $\left.d=\frac{3}{2} \pi-\frac{3}{2} \phi(k, \ell)+\mathbb{Z} 3 \pi\right)$.

\begin{tabular}{|c|c|c|c|}
\hline$k$ & $\ell$ & $\phi$ & $d$ \\
\hline 0 & 1 & $\frac{1}{6} \pi$ & $\frac{5}{4} \pi$ \\
\hline 1 & 0 & $\frac{7}{6} \pi$ & $\frac{11}{4} \pi$ \\
\hline 0 & 2 & $\frac{11}{6} \pi$ & $\frac{7}{4} \pi$ \\
\hline 2 & 0 & $\frac{5}{6} \pi$ & $\frac{1}{4} \pi$ \\
\hline 1 & 2 & $\frac{9}{6} \pi$ & $\frac{9}{4} \pi$ \\
\hline 2 & 1 & $\frac{3}{6} \pi$ & $\frac{3}{4} \pi$ \\
\hline
\end{tabular}

The analytic data consists of the formal monodromy and the six Stokes matrices at $z=\infty$. The product of the formal monodromy and the Stokes matrices for the singular directions $d \in[0,2 \pi)$

$$
\left(\begin{array}{lll}
0 & 0 & 1 \\
1 & 0 & 0 \\
0 & 1 & 0
\end{array}\right)\left(\begin{array}{lll}
1 & 0 & 0 \\
0 & 1 & 0 \\
x_{4} & 0 & 1
\end{array}\right)\left(\begin{array}{lll}
1 & 0 & 0 \\
x_{3} & 1 & 0 \\
0 & 0 & 1
\end{array}\right)\left(\begin{array}{lll}
1 & 0 & 0 \\
0 & 1 & x_{2} \\
0 & 0 & 1
\end{array}\right)\left(\begin{array}{lll}
1 & 0 & x_{1} \\
0 & 1 & 0 \\
0 & 0 & 1
\end{array}\right)
$$

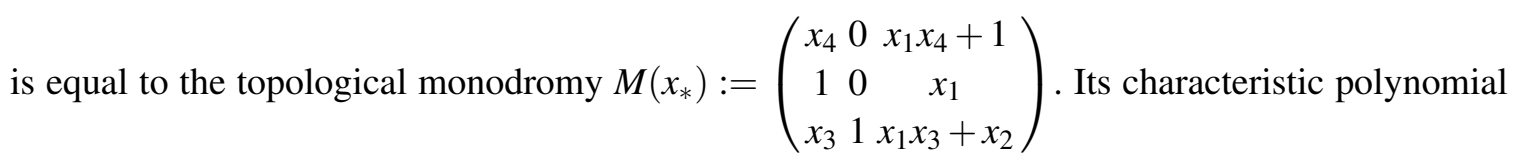
is $\lambda^{3}-\left(x_{2}+x_{4}+x_{1} x_{3}\right) \lambda^{2}+\left(-x_{1}-x_{3}+x_{2} x_{4}\right) \lambda-1$.

The moduli space $\mathscr{R}$ for the analytic data consists of the tuples $x_{*}=\left(x_{1}, \ldots, x_{4}\right)$ since the other two Stokes matrices can be expressed in the Stokes matrices for the singular directions in $[0,2 \pi)$. Thus $\mathscr{R} \cong \mathbb{A}^{4}$. The elements of the parameter space $\mathscr{P}$ are the sets of eigenvalues of the topological monodromy. Thus $\mathscr{P}=\left\{\lambda^{3}-e_{1} \lambda^{2}+e_{2} \lambda-1 \mid e_{1}, e_{2} \in \mathbb{C}\right\}$. Let $\mathscr{R}(P)$ be the fibre above $P \in \mathscr{P}$. If $P$ has three distinct roots, then $\mathscr{R}(P)$ is a smooth surface. If $P$ has roots 
$a, a, a^{-2}$ with $a \neq a^{-2}$, then the point $x_{*} \in \mathscr{R}(P)$ with $x_{*} \neq\left(-a^{-1}, a,-a^{-1}, a\right)$ is regular and $M\left(x_{*}\right)$ has two Jordan blocks. The point $\left(-a^{-1}, a,-a^{-1}, a\right) \in \mathscr{R}(P)$ is singular and has type $A_{1}$. Further $M\left(-a^{-1}, a,-a^{-1}, a\right)$ has three Jordan blocks. If $P$ has roots $a, a, a$ (and thus $\left.a^{3}=1\right)$, then the point $x_{*} \in \mathscr{R}(P)$ with $x_{*} \neq\left(-a^{-1}, a,-a^{-1}, a\right)$ is regular and $M\left(x_{*}\right)$ has one Jordan block. The point $x_{*}=\left(-a^{-1}, a,-a^{-1}, a\right)$ is singular and has type $A_{2}$. The matrix $M\left(-a^{-1}, a,-a^{-1}, a\right)$ has two Jordan blocks.

Level structure for $\mathscr{R}$ and $\mathscr{P}$. For an element of $\mathscr{R}$ we introduce a 'level structure' which consists of subspaces $L_{1} \subset L_{2} \subset \mathbb{C}^{3}$ of dimensions 1 and 2 which are invariant under the topological monodromy (at $z=\infty$ or, equivalently, at $z=0$ ). The corresponding space is denoted by $\mathscr{R}^{+}$. The level structure for a $P \in \mathscr{P}$ consists of a tuple $\left(\mu_{1}, \mu_{2}, \mu_{3}\right)$ with $\mu_{1} \mu_{2} \mu_{3}=1$ and $P=\prod_{j=1}^{3}\left(\lambda-\mu_{j}\right)$. The corresponding space is denoted by $\mathscr{P}^{+}$. The morphism par: $\mathscr{R}^{+} \rightarrow \mathscr{P}^{+}$is defined by $\left(\left(x_{*}\right), L_{1}, L_{2}\right) \mapsto\left(\mu_{1}, \mu_{2}, \mu_{3}\right)$, where $\mu_{1}$ is the eigenvalue of $M\left(x_{*}\right)$ on $L_{1}$ and $\mu_{2}$ is that of $M\left(x_{*}\right)$ on $L_{2} / L_{1}$.

One observes that $\mathscr{R}^{+}$is the closed subspace of $\mathbb{C}_{x_{*}}^{4} \times \mathbb{P}_{y_{*}}^{2} \times\left(\mathbb{P}^{2}\right)_{z_{*}}^{*} \times \mathscr{P}^{+}$, where $\mathscr{P}+$ equals $\left\{\left(\mu_{1}, \mu_{2}, \mu_{3}\right) \in \mathbb{C}^{3} \mid \mu_{1} \mu_{2} \mu_{3}=1\right\}$, given by the equations:

$M\left(x_{*}\right) y=\mu_{1} y, y:=\left(\begin{array}{l}y_{1} \\ y_{2} \\ y_{3}\end{array}\right), z M\left(x_{*}\right)=\mu_{3} z, z:=\left(z_{1}, z_{2}, z_{3}\right), \sum y_{j} z_{j}=0$.

Indeed, $\mathbb{C} y$ and the kernel of $z \in\left(\mathbb{C}^{3}\right)^{*}$ are the $M\left(x_{*}\right)$-invariant spaces $L_{1} \subset L_{2} \subset \mathbb{C}^{3}$. Further par: $\mathscr{R}^{+} \rightarrow \mathscr{P}^{+}$is the projection onto the last factor. The fibre $\mathscr{R}^{+}\left(\mu_{*}\right)$ of par above the point $\left(\mu_{*}\right) \in$ $\mathscr{P}^{+}$maps to the fibre $\mathscr{R}(P)$ of $\mathscr{R} \rightarrow \mathscr{P}$ above the point $P=\left(\lambda-\mu_{1}\right)\left(\lambda-\mu_{2}\right)\left(\lambda-\mu_{3}\right)$.

Proposition 3.1. res : $\mathscr{R}^{+}\left(\mu_{*}\right) \rightarrow \mathscr{R}(P)$ is the minimal resolution of $\mathscr{R}(P)$.

A straightforward computation proves this statement. In particular, the fibre of res above a non singular point is just one point since there is only one level structure possible. If two of the $\mu_{*}$ are equal, then the preimage under res of the singular point is a $\mathbb{P}^{1}$, consisting of the lines $\mathbb{C} y$ in the two-dimensional eigenspace for $a$ and the kernel of $z$ is this two-dimensional eigenspace. If the three $\mu_{*}$ are equal, then the preimage under res of the singular point is a pair of intersecting projective lines. In this case the Jordan form of $M\left(x_{*}\right)$ has two blocks, $\mathbb{C} y$ is a line in the two-dimensional eigenspace of $\mathbb{C}^{3}, \mathbb{C} z$ is a line in the two-dimensional eigenspace of the dual $\left(\mathbb{C}^{3}\right)^{*}$ and $\sum y_{j} z_{j}=0$.

Proposition 3.2. The natural maps $\mathbf{S} \rightarrow \mathscr{R} \times T$ and $\mathbf{S}^{+} \rightarrow \mathscr{R}^{+} \times T$ (with $T=\mathbb{C}$ ) are bijections.

Proof. In the first case one applies [vdP-Saito], Theorem 1.7. The second case follows from the observation that the level structure $M_{1} \subset M_{2} \subset \mathbb{C}((z)) \otimes M$ induces subspaces $L_{1} \subset L_{2} \subset \mathbb{C}^{3}$ of dimensions 1 and 2, invariant under the topological monodromy, and visa versa.

The Noumi-Yamada moduli space $\mathscr{N}^{+}\left(\varepsilon_{*}\right)$.

$\varepsilon_{*}$ denotes a triple $\left(\varepsilon_{1}, \varepsilon_{2}, \varepsilon_{3}\right)$ with $\sum \varepsilon_{j}=0$. The set $\mathbf{S}^{+}\left(\varepsilon_{*}\right)$ consists of the tuples $\left(M, M_{1} \subset M_{2}\right) \in \mathbf{S}^{+}$ such that $M_{1}=\mathbb{C}((z)) b_{1}$ with $\delta_{M}\left(b_{1}\right)=\varepsilon_{1} b_{1}$ and $M_{2} / M_{1}=\mathbb{C}((z)) b_{2}$ with $\delta_{M}\left(b_{2}\right)=\varepsilon_{2} b_{2}$. Let $\mathscr{V}$ denote the free bundle on $\mathbb{P}^{1}$ of rank 3.

The points of the moduli space $\mathscr{N}^{+}\left(\varepsilon_{*}\right)$ correspond to the isomorphy classes of connections $D:=\nabla_{z_{d z}}: \mathscr{V} \rightarrow O([\infty]) \otimes \mathscr{V}$ with a level structure which consists of the $D$-invariant submodules $V_{1} \subset V_{2} \subset \widehat{\mathscr{V}}_{0}$ of rank 1 and 2 such that $\widehat{\mathscr{V}}_{0} / V_{j}, j=1,2$ have no torsion and such that there is a tuple 
$\left(M, M_{1} \subset M_{2}\right) \in \mathbf{S}^{+}\left(\varepsilon_{*}\right)$ with $M$ is the generic fibre of $\mathscr{V}, \mathbb{C}((z)) \otimes V_{j}=M_{j}, j=1,2$ and $\widehat{\mathscr{V}}_{\infty}$ is the lattice $\Lambda_{0} \subset \mathbb{C}\left(\left(z^{-1}\right)\right) \otimes M$.

Proposition 3.3. $\mathscr{N}^{+}\left(\varepsilon_{*}\right)$ is the affine space $\mathbb{A}^{3}$ with coordinates $f_{0}, f_{1}, f_{2}, t=f_{0}+f_{1}+f_{2}$ and the connection is represented by $z \frac{d}{d z}+\left(\begin{array}{ccc}\varepsilon_{1} & f_{1} & 1 \\ z & \varepsilon_{2} & f_{2} \\ f_{0} z & z & \varepsilon_{3}\end{array}\right)$.

Proof. The level structure provides $H^{0}(\mathscr{V})$ with a basis $e_{1}, e_{2}, e_{3}$ such that $D=z \frac{d}{d z}+A_{0}+A_{1} z$ with traceless constant matrices $A_{0}, A_{1}$ and $A_{0}=\left(\begin{array}{ccc}\varepsilon_{1} & * & * \\ 0 & \varepsilon_{2} & * \\ 0 & 0 & \varepsilon_{3}\end{array}\right)$. This is unique up to the action

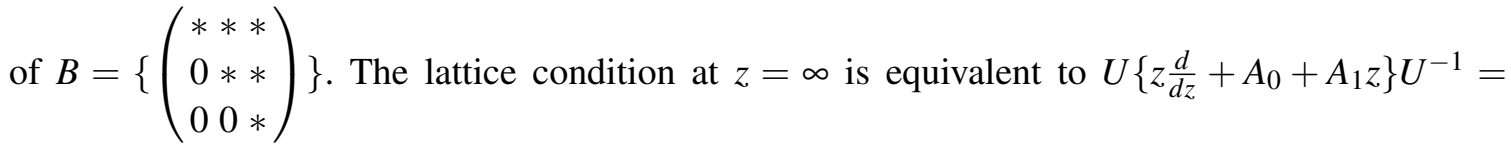
$z \frac{d}{d z}+\left(\begin{array}{ccc}0 & z & \frac{t}{3} \\ \frac{t}{3} & \frac{1}{3} & 1 \\ z & \frac{t}{3} z & -\frac{1}{3}\end{array}\right)$ for some $U=U_{0}\left(1+U_{-1} z^{-1}+\ldots\right) \in \mathrm{GL}_{3}\left(\mathbb{C}\left[\left[z^{-1}\right]\right]\right)$. This is equivalent to the equations $A_{1}=U_{0}^{-1}\left(\begin{array}{lll}0 & 1 & 0 \\ 0 & 0 & 0 \\ 1 & \frac{t}{3} & 0\end{array}\right) U_{0}$ and $A_{0}=U_{0}^{-1}\left(\begin{array}{ccc}0 & 0 & \frac{t}{3} \\ \frac{t}{3} & \frac{1}{3} & 1 \\ 0 & 0 & -\frac{1}{3}\end{array}\right) U_{0}+\left[A_{1}, U_{-1}\right]$. A MAPLE computation produces matrices $U_{0}$ and $U_{-1}$ such that $A_{0}=\left(\begin{array}{ccc}\varepsilon_{1} & * & * \\ 0 & \varepsilon_{2} & * \\ 0 & 0 & \varepsilon_{3}\end{array}\right)$ and $A_{1}=\left(\begin{array}{ccc}0 & 0 & 0 \\ 1 & 0 & 0 \\ * & 1 & 0\end{array}\right)$. Moreover $U_{0}$ is unique up to multiplication by a non zero constant and $U_{-1}$ is unique up to adding a matrix $V$ with $\left[A_{1}, V\right]=0$. Thus we found a representation of the connection in the 'Noumi-Yamada form' and this form is unique with respect to the action of the Borel group $B$ on $\mathscr{V}$. Therefore the Noumi-Yamada form represents the moduli space $\mathscr{N}^{+}\left(\varepsilon_{*}\right)$.

The map $\mathscr{N}\left(\varepsilon_{*}\right) \rightarrow \mathbf{S}^{+}\left(\varepsilon_{*}\right)$ is injective and not bijective. This is due to the choice of a free vector bundle $\mathscr{V}$ in the construction of $\mathscr{N}\left(\varepsilon_{*}\right)$. The aim is to avoid this choice and to construct a smooth partial completion $\widehat{\mathscr{N}}\left(\varepsilon_{*}\right)$ such that $\widehat{\mathscr{N}}\left(\varepsilon_{*}\right) \rightarrow \mathbf{S}^{+}\left(\varepsilon_{*}\right)$ is bijective. As in $\S 2$, this will imply that the extended Riemann-Hilbert map $\widehat{\mathscr{N}}\left(\varepsilon_{*}\right) \rightarrow \mathscr{R}^{+}\left(\mu_{*}\right) \times T$ (with $\mu_{j}=e^{2 \pi i \varepsilon_{j}}$ for $j=1,2,3$ ) is an analytic isomorphism. Moreover $\widehat{\mathscr{N}}\left(\varepsilon_{*}\right)$ is the Okamoto-Painlevé space and $\mathscr{R}^{+}\left(\mu_{*}\right)$ is the space of the initial conditions.

Construction of $\widehat{\mathscr{N}}\left(\varepsilon_{*}\right)$.

The points of $\widehat{\mathscr{N}}\left(\varepsilon_{*}\right)$ correspond to (the isomorphism classes of) the tuples $\left(\mathscr{V}, D, L_{1}, L_{2}\right)$ with $D=\nabla_{z \frac{d}{d z}}: \mathscr{V} \rightarrow O([\infty]) \otimes \mathscr{V}$ is a connection on a vector bundle $\mathscr{V}$ of rank 3.

We require the following: The connection $\widehat{\mathscr{V}}_{\infty}$ is isomorphic to $\Lambda_{1}$. In other terms $\widehat{\mathscr{V}}_{\infty}$ has a basis over $\mathbb{C}\left[\left[z^{-1}\right]\right]$ for which $D$ has the form $z \frac{d}{d z}+\left(\begin{array}{ccc}0 & 1 & \frac{t}{3} \\ \frac{t}{3} z & -\frac{2}{3} & z \\ z & \frac{t}{3} & -\frac{1}{3}\end{array}\right)$. Further $L_{1}=\mathbb{C}[[z]] Y$ is a subconnection of $\widehat{\mathscr{V}}_{0}$ such that $\widehat{\mathscr{V}}_{0} / L_{1}$ has no torsion and $D Y=\varepsilon_{1} Y$. Further $L_{2}=\mathbb{C}[[z]] Z$ is a subconnection of $\widehat{\mathscr{V}}_{0}^{*}$, the dual of $\widehat{\mathscr{V}}_{0}$, such that $\widehat{\mathscr{V}}_{0}^{*} / L_{2}$ has no torsion and $D Z=\varepsilon_{3} Z$. Moreover $\Lambda^{3}\left(\widehat{\mathscr{V}}_{0}\right)$ is trivial and 
$L_{2}\left(L_{1}\right)=0$.

The map $F: \widehat{\mathscr{N}}\left(\varepsilon_{*}\right) \rightarrow \mathbf{S}^{+}\left(\varepsilon_{*}\right)$, sends $\left(\mathscr{V}, D, L_{1}, L_{2}\right)$ to its generic fibre $M$ together with the level structure on $\mathbb{C}((z)) \otimes M$ obtained from $L_{1}, L_{2}$. Conversely, for a given element $\left(M, M_{1} \subset M_{2}\right) \in$ $\mathbf{S}^{+}\left(\varepsilon_{*}\right)$ one defines the connection $(\mathscr{V}, D)$ with generic fibre $M$, by prescribing $\widehat{\mathscr{V}}_{\infty} \cong \Lambda_{1}$. The additional data $L_{1}, L_{2}$ imply that $\widehat{\mathscr{V}}_{0}$ is represented by $z \frac{d}{d z}+\left(\begin{array}{ccc}\varepsilon_{1} & * & * \\ 0 & \varepsilon_{2} & * \\ 0 & 0 & \varepsilon_{3}\end{array}\right)$ with all $* \in \mathbb{C}[[z]]$. This implies that $F$ is bijective in the following cases:

$$
\begin{aligned}
& \mu_{1}, \mu_{2}, \mu_{3} \text { are distinct; } \\
& \mu_{1}=\mu_{2} \neq \mu_{3} \text { and } \varepsilon_{2}-\varepsilon_{1} \geq 0 ; \\
& \mu_{1}=\mu_{3} \neq \mu_{2} \text { and } \varepsilon_{3}-\varepsilon_{1} \geq 0 ; \\
& \mu_{1} \neq \mu_{2}=\mu_{3} \text { and } \varepsilon_{3}-\varepsilon_{2} \geq 0 ; \\
& \mu_{1}=\mu_{2}=\mu_{3} \text { and } \varepsilon_{2}-\varepsilon_{1}, \varepsilon_{3}-\varepsilon_{2} \geq 0 .
\end{aligned}
$$

In the sequel we will only consider these cases.

In order to give $\widehat{\mathscr{N}}\left(\varepsilon_{*}\right)$ the structure of an algebraic variety we observe that $\mathscr{V}$ has degree -1 and type $O \oplus O \oplus O(-1)$ since $(\mathscr{V}, D)$ is irreducible. We identify $\mathscr{V}$ with $O e_{1} \oplus O e_{2} \oplus$ $O(-[\infty]) e_{3}$. The matrix of $D$ with respect to the basis $e_{1}, e_{2}, e_{3}$ has trace zero and is denoted by

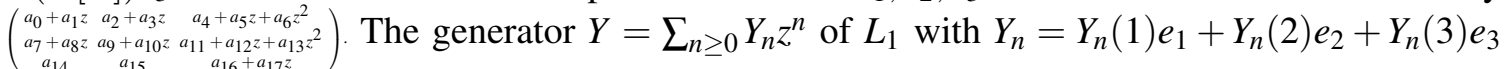
is unique up to multiplication by a constant. The generator $Z=\sum_{n \geq 0} Z_{n} z^{n}$ of $L_{2}$ with $Z_{n}=$ $Z_{n}(1) e_{1}^{*}+Z_{n}(2) e_{2}^{*}+Z_{n}(3) e_{3}^{*}$ is unique up to multiplication by a constant. The $Y_{*}(*), Z_{*}(*)$ are regarded as homogeneous coordinates.

The space $\mathscr{A}$ is defined by the indeterminates $a_{*}, Y_{*}(*), Z_{*}(*)$ and the relations induced by the above requirements. We note that for given $\varepsilon_{*}$, such that the above restrictions are satisfied, the $Y_{n}(*), Z_{n}(*)$ are for $n \geq 1$ eliminated by the relations. Thus $\mathscr{A}$ is an algebraic variety.

The group $G$ of the automorphisms of $\mathscr{V}$ act upon $\mathscr{A}$. By construction, the set theoretic quotient $\mathscr{A}(\mathbb{C}) / G$ coincides with $\mathbf{S}^{+}\left(\varepsilon_{*}\right)$. Thus the analytic map $R: \mathscr{A} \rightarrow \mathscr{R}^{+}\left(\mu_{*}\right) \times T$, where $\mu_{j}=e^{2 \pi i \varepsilon_{j}}$ for $j=1,2,3$, is surjective and $R\left(\xi_{1}\right)=R\left(\xi_{2}\right)$ if and only if there is a $g \in G$ with $g \xi_{1}=\xi_{2}$.

A long MAPLE session verifies: $\mathscr{A}$ has a smooth geometric quotient by $G$. This quotient is by definition $\widehat{N}\left(\varepsilon_{*}\right)$ and the extended Riemann-Hilbert map $\widehat{N}\left(\varepsilon_{*}\right) \rightarrow \mathscr{R}^{+}\left(\mu_{*}\right) \times T$ is an analytic isomorphism.

\section{References}

[Bo] P. Boalch, Symplectic Manifolds and Isomonodromic Deformations, Adv. in Math. 163 (2001) 137-205.

[Gr] V.I. Gromak, I. Laine, S. Shimomura, Painleve differential equations in the complex plane. de Gruyter Studies in Mathematics, 28. Walter de Gruyter \& Co., Berlin, 2002.

[In] M. Inaba, Moduli of parabolic connections on a curve and Riemann-Hilbert correspondence, Preprint, 2006, arXiv:math/0602004.

[IIS1] M. Inaba, K. Iwasaki, and M.-H. Saito, Moduli of stable parabolic connections, Riemann-Hilbert correspondence and geometry of Painlevé equation of type VI. I, Publ. Res. Inst. Math. Sci. 42 (2006) no. 4 987-1089.

[IIS2] M. Inaba, K. Iwasaki, and M.-H. Saito, Moduli of stable parabolic connections, Riemann-Hilbert correspondence and geometry of Painlevé equation of type VI. II, Moduli spaces and arithmetic geometry (Tokyo), Adv. Stud. Pure Math., vol. 45, Math. Soc. Japan, Tokyo, 2006, pp. 387-432. 
[IISA] M. Inaba, K. Iwasaki and M.-H. Saito, Dynamics of the sixth Painlevé Equations, Théories Asymptotiques et Équations de Painlevé, Angers, Juin, 2004, "Séminaires et Congrès" of the Société Mathématique de France (SMF)14, 2006, 103-167.

[JMU] M. Jimbo, T. Miwa and K. Ueno, Monodromy preserving deformation of linear ordinary differential equations with rational coefficients. I. General theory and $\tau$-function, Physica D 2 (1981) 306-352.

[JM] M. Jimbo and T. Miwa, Monodromy preserving deformation of linear ordinary differential equations with rational coefficients. II., Physica D 2 (1981) 407-448.

[No] M. Noumi, Painlevé equations through symmetry. Translated from the 2000 Japanese original by the author. Translations of Mathematical Monographs, 223. American Mathematical Society, Providence, RI, 2004.

[No-Y] M. Noumi and Y. Yamada, Symmetries in the fourth Painlevé equation and Okamoto polynomials. Nagoya Math. J. 153 (1999) 53-86.

[OO] Y. Ohyama, S. Okumura, A coalescent diagram of the Painlevé equations from the viewpoint of isomonodromic deformations. J. Phys. A 39 (2006) no. 39 12129-12151.

[O1] K. Okamoto, Sur les feuilletages associés aux équations du second ordre à points critiques fixes de P. Painlevé, Espaces des conditions initiales, Japan. J. Math. 5 (1979) 1-79.

[O2] K. Okamoto, Isomonodromic deformation and Painlevé equations and the Garnier system, J. Fac. Sci. Univ. Tokyo, Sect. IA, Math. 33 (1986) 575-618.

[O3] K. Okamoto, Studies on the Painlevé Equations III Second and Fourth Painlevé Equations, $P_{I I}$ and $P_{I V}$, Math. Ann. 275 (1986) 221-255.

[O4] K. Okamoto, The Hamiltonians associated to the Painlevé equations. The Painlevé property, 735787, CRM Ser. Math. Phys. Springer, New York, 1999.

[vdP1] M. van der Put, Families of linear differential equations and the Painlevé equations, $S M F$, Séminaires \& Congrès 27 (2012) 203-220.

[vdP2] M. van der Put, Families of linear differential equations related to the second Painlevé equation in: Algebraic Methods in Dynamical Systems, Banach Center Publications, Volume 94, 2011, 247-262.

[vdP-Sa] M. van der Put and M-H. Saito, Moduli spaces for linear differential equations and the Painlevé equations., Ann. Inst. Fourier 59 (2009) no. 7 2611-2667.

[vdP-Si] M. van der Put, M.F. Singer, Galois Theory of Linear Differential Equations, Grundlehren der mathematischen Wissenschaften, Volume 328, Springer Verlag 2003.

[STT] M.-H. Saito, T. Takebe, H. Terajima, Deformation of Okamoto-Painlevé pairs and Painlevé equations, J. Algebraic Geom. 11 (2002) no. 2 311-362.

[S-Ta] Saito, M-H., Takebe, T., Classification of Okamoto-Painlevé pairs, Kobe J. Math. 19 (2002) no. 1-2 21-50.

[SU] M.-H. Saito, H. Umemura, Painlevé equations and deformations of rational surfaces with rational double points. Physics and combinatorics 1999 (Nagoya), 320-365, World Sci. Publishing, River Edge, NJ, 2001.

[STe] M.-H. Saito, H. Terajima, Nodal curves and Riccati solutions of Painlevé equations, J. Math. Kyoto Univ. 44 (2004) no. 3 529-568.

[Sakai] H. Sakai, Rational surfaces associated with affine root systems and geometry of the Painlevé equations, Comm. Math. Phys. 220 (2001) 165-229.

[T] H. Terajima, Families of Okamoto-Painlevé pairs and Painlevé equations, Ann. Mat. Pura Appl. (4), 186 (2007) no. 1 99-146. 